\title{
SK-ER3 Neuroblastoma Cells as a Model for the Study of Estrogen Influence on Neural Cells
}

\author{
PAOLA AGRATI, MARTINE GARNIER, CESARE PATRONE, GIUSEPPE POLLIO, SABRINA SANTAGATI, \\ ELISABETTA VEGETO AND ADRIANA MAGGI ${ }^{1}$
}

Center MPL, Institute of Pharmacological Sciences, University of Milan, Via Balzaretti 9, 20133, Milan, Italy

\begin{abstract}
The neuroblastoma SK-ER3 cell line obtained by stable transfection of the human SK-N-BE cell line is proposed as a model for the study of estrogen receptor activity in cells of neural origin. In the SK-ER3 cell line the estrogen receptor, once activated, initiates a differentiation program leading to growth arrest, morphological changes, and acquisition of the dopaminergic phenotype. In the absence of estrogens, this program can be triggered by IGF-I, which can activate the unliganded estrogen receptor via the ras-pathway. It is proposed that this model system might recapitulate the events occurring in vivo during the differentiation of the nervous system and that IGF-I may play an important role in the activation of estrogen receptor at the very early stage of brain development affecting the differentiation of a number of hypothalamic and extrahypothalamic brain regions. () 1997 Elsevier Science Inc.
\end{abstract}

KEY WORDS: Neuroblastoma, Stable transfection, Estrogen receptor, Differentiation, Brain.

\section{INTRODUCTION}

Estradiol is a well-known differentiating agent covering a fundamental role in the development of reproductive and non reproductive tissues. In fact, it has been long known that the maturation of chicken oviduct cells, mammalian uterine tissue, or frog hepatocytes is dependent upon estrogen priming [13]. On the basis of these studies and as suggested by more recent work carried out on ecdysone [19], another steroid endowed of differentiating potential in insects, it could be postulated that estradiol starts the differentiation of target, still multipotent blast cells, by inducing a series of early genes, which in turn, would provoke a cascade of events resulting in the activation of all the genes necessary for the final, permanent, phenotype of the estrogen receptor-expressing cells (e.g., for oviduct, differentiation is paralleled by the expression of the progesterone receptor together with all the other proteins typical of the mature cell like ovomucoid, ovalbumine, etc.).

The nervous system can be considered among the tissues target for the estrogen differentiating activity. The current view of brain sexual differentiation, in fact, suggests that, in the first few days of life, the intracellular aromatization of the testosterone synthesized by the gonads to $17 \beta$-estradiol $\left(\mathrm{E}_{2}\right)$, which subsequently binds to high-affinity estrogen receptors (ER), causes the acquisition of those neural circuits, which in the adult animal will allow the display of the male sexual behavior. In the absence of estrogens, the adult animal will display a female-like behavior, independently of the presence of the male sex chromosome in its genome $[2,5,15]$.

However, several localization studies prove that, during the maturation of the nervous system, ERs are expressed in several brain areas much earlier than expected on the basis of the theory mentioned above. For instance in rat brain, ER appears in the basal hypothalamus, preoptic area, amygdala, mid brain and spinal cord on day E14. The number of ER-expressing cells increases in each of these areas on days E15, E17, and P0 [6,11]. Also, cells of the peripheral nervous system express ER, as reported by Sohrabji et al. [17]. In some of the above-mentioned brain areas (e.g., the hippocampus [12], the levels of ER increase during the late embryonic phase to rapidly decrease at birth, that is, at the time in which the estrogen-induced differentiation of neural cells should occur. It is worth underlining that the current belief on the events triggering brain sexual differentiation regards the embryonal expression of the ER as deprived of any functional meaning. In fact, it is known that the maternal estradiol cannot activate the embryo receptors due to the presence of $\alpha$-fetoprotein, which ties up the circulating hormone and prevents its binding to the ER. The aromatizable testerone, on the other hand, is produced only perinatally. Nevertheless, studies carried out in cultures of dispersed embryo neural cells demonstrate that estradiol may interact with the expressed ER to influence the cell physiology and prove that the ER, expressed in embryonal neural cells, are fully functional $[4,16,18]$. On the basis of these findings it could be hypothesized that ER might have an effect on the earlier phases of the nervous system development. Further experiments should be undertaken to prove this hypothesis. However, the use of cultures of dispersed embryonal cells have a number of drawbacks limiting their use: the majority of which is represented by the fact that only a minute percentage of the neural cells expresses the ER.

The goal of our studies was to set up a cell system for the study of ER activity in cells of neural origin.

\section{METHODS}

\section{Neuroblastoma Cell Growth and Transfection}

SK-N-BE and SK-ER3 cells were routinely cultured in RPMI1640 medium without phenol red (Sigma) and supplemented with $10 \%$ charcoal stripped fetal bovine serum (DCC-FBS). For transfection studies, $24 \mathrm{~h}$ before transfection, $4 \times 10^{5}$ cells were plated in six-well dishes containing $3 \mathrm{ml}$ phenol red-free RPMI 1640 medium supplemented with $10 \%$ DCC-FBS. Six hours before addition of the $\mathrm{CaPO}_{4} / \mathrm{DNA}$ mixture, the medium was replaced

\footnotetext{
${ }^{1}$ To whom requests for reprints should be addressed.
} 

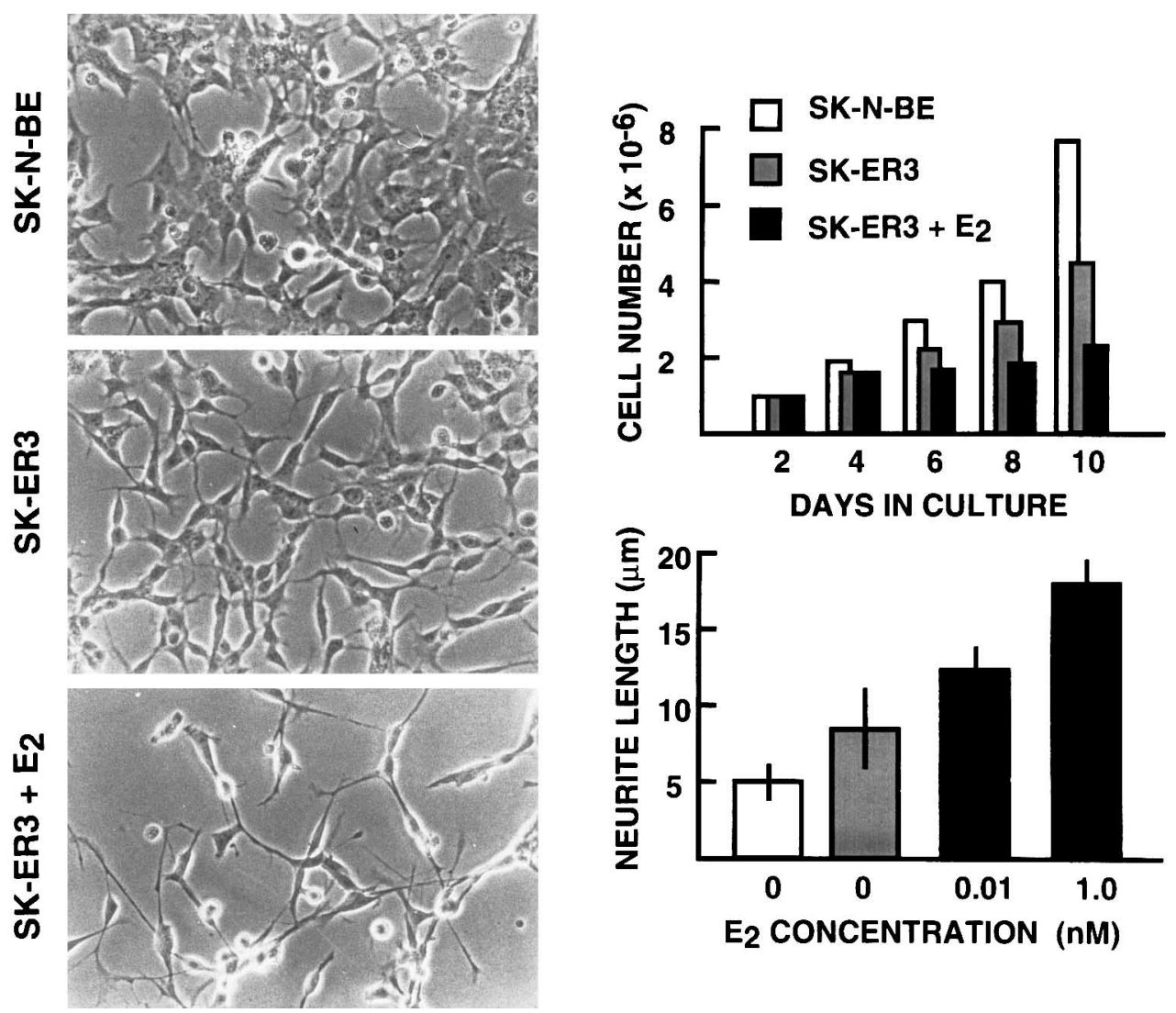

FIG. 1. Left: contrast phase photograph of SK-N-BE and SK-ER3 cells $\pm 1 \mathrm{nM} \mathrm{E} \mathrm{E}_{2}$ for 10 days $(\times 130$; Nikon microscope). Right: upper panel: cells were plated in duplicate and counted at the indicated day by trypan blue exclusion in a Burker chamber. The data reported represent the avarage of three separate experiments. Lower panel: SK-N-BE (open bars) and SK-ER3 (filled bars) cells were grown for 12 days, then fixed and analyzed as reported in the methods section. Black bars refer to SK-ER3 cells treated with $1 \mathrm{nM}$ estradiol for the entire lenght of the experiment. Bars represent the SD of a minimum of 150 measurements/experimental group carried out on cells grown in three separate experiments.

with DMEM containing 10\% DCC-FBS. In a typical transient transfection experiment, the cells were transfected with $200 \mu \mathrm{l}$ of a suspension containing $0.1 \mu \mathrm{g} / \mathrm{ml}$ of ERcDNA, $0.05 \mu \mathrm{g} / \mathrm{ml}$ of the reporter gene (pVEREt-kCAT), $0.1 \mu \mathrm{g} / \mathrm{ml}$ of $\mathrm{p} 21$ (asn17) ras, and $0.12 \mu \mathrm{g} / \mathrm{ml}$ of carrier DNA (pGEM3z) in $1.8 \mathrm{ml}$ of medium (in the stable transfection the reporter gene was omitted) [9]. Sixteen hours after the addition of the precipitate, the medium was discarded and, after a few washes with RPMI 1640, replaced with phenol red-free RPMI 1640 containing 1\% DCC-FBS. In transient transfections, the pharmacological treatments were started at this point. Twenty-four hours later the medium was removed, the cells were washed several times with PBS, and cellular extracts were prepared following the protocol of the CAT-ELISA kit (Boehringer M., Milan, Italy). The protein content was measured by Bradford's assay. Each experiment was repeated at least four times.

For stably transfected cells, the selection was done in the presence of 300-400 $\mu \mathrm{g}$ of geneticine/ml. The surviving clones were then amplified and analyzed for ER expression [9].

\section{Cell Proliferation Studies}

Cells $\left(1.5 \times 10^{4}\right)$ were plated in triplicate in $25-\mathrm{cm}^{2}$ flasks. Cell number and viability in the presence or absence of $E_{2}(1 \mathrm{nM})$ was evaluated using $0.4 \%$ trypan blue stain (Sigma) according to the technical instructions.

\section{${ }^{3} H$ Thymidine Incorporation Assay}

${ }^{3} \mathrm{H}$-thymidine incorporation was estimated by plating $10^{4}$ cells in a six-well dish. The cells were grown for 12 days following the hormonal treatment. ${ }^{3} \mathrm{H}$-thymidine (Amersham, $1.0 \mu \mathrm{Ci} / \mathrm{ml} \mathrm{me-}$ dium) was added $3 \mathrm{~h}$ before harvesting the cells. Cells were lysed and assayed for radioactivity by addition of scintillation fluid.

\section{Morphometric Analysis}

SK-ER3 cells were grown on coverslips pretreated with $5 \%$ gelatin in the presence or absence of $E_{2}(1 \mathrm{nM})$. Cells were then fixed at room temperature with $4 \%$ paraformaldehyde in $200 \mathrm{mM}$ borate buffer for $10 \mathrm{~min}$. After a few rinses the cells were incubated for $5 \mathrm{~min}$ in the presence of $2 \%$ horse serum and $0.1 \%$ Triton $\mathrm{X}-100$ in PBS. Anti-Tau (ICN) and antisynaptophysin (Boehringer-Mannheim) at 1:100 and 1:5 dilutions, respectively, were applied and incubated overnight at $4{ }^{\circ} \mathrm{C}$ in a humidified chamber. After several rinses in PBS, cells were incubated with a 1:100 dilution of a fluorescein-conjugated horse antimouse secondary antibody (Vector Laboratories) for $2 \mathrm{~h}$ at room temperature. After 
TABLE 1

SK-ER3 MUST BE TREATED WITH E, FOR A MINIMUM TIME LENGTH OF 16 HOURS TO STOP PROLIFERATION

$\mathrm{E}_{2}$ Treatment (Length) ${ }^{3} \mathrm{H}$ Thymidine Incorporation (at the 12th Day in Culture)

$\begin{array}{lc}\text { Controls } & 4,700 \pm 140 \\ 10 \mathrm{~min} & 2,200 \pm 260 \\ 30 \mathrm{~min} & 1,900 \pm 220 \\ 1 \mathrm{~h} & 1,600 \pm 50 \\ 4 \mathrm{~h} & 1,900 \pm 110 \\ 16 \mathrm{~h} & 0.02 \pm 5 \\ 12 \text { days } & 0.02 \pm 6\end{array}$

Cells were treated with estrogens for the indicated time, then the medium was removed and replaced with fresh medium deprived of steroids. Cells were grown for 12 more days then the ${ }^{3} \mathrm{H}$ thymidine incorporation assay was performed in triplicate.

several washes, coverslips were mounted onto slides using PermaFluor (Helena Laboratories). Main neuritic length of individual neurons was quantified using an automatic image analyzer equipped with IMAGE software developed and generously provided by Wayne Rosband (National Institutes of Health, Bethesda, MD). A total of 60 cells were analyzed for each experimental group. Statistical analysis was performed using ANOVA. $p \leq 0.05$ was taken as the level of statistical significance.

\section{RESULTS}

Ligand-Operated Activation of ER Causes Growth Arrest and Differentiation of SK-ER3 Neuroblastoma Cells

To set up a system for the study of the effects of estrogens on neural cells we engineered a neuroblastoma cell line by stable transfection of the ER cDNA under the control of a constitutive promoter. As parental neuroblastoma we selected a cell line representing an early stage of neural crest cell differentiation. Cells of the human line SK-N-BE, in fact, have been reported to interconvert between two cell phenotypes: neural and melanocytic, which are known to represent the very initial stage of the differentiation of neural crest cells. The theoretical limitation of the model we chose is that neuroblastoma cells might express a number of genes conferring them the tumoral, proliferative phenotype, which might mask or override the expression of genes characterising a neural cell; furthermore, in these cells the expression of the ER is due to the addition of an heterologous gene that might not be subjected to the natural regulatory mechanisms. On the other hand, we thought that this model would have facilitated the identification of the genes target of the ER receptor and would have been amenable to a number of pharmacological and biochemical studies that "physiological relevance" could then be evaluated either in vivo or in primary culture cells.

In the cell line we generated and named SK-ER3, as shown in Fig. 1, the activation of the ER results in morphological alterations and growth arrest quantitated by measurement of the neurite length and cell counting, respectively. Subsequent experiments by cell counting (Table 1) and analysis of tritiated thymidine incorporation (not shown) demonstrated that the exposure to the hormone must be prolonged for at least $16 \mathrm{~h}$ to commit the cells to the proliferation arrest. After this time period, the hormone can be removed from the medium without affecting the growth arrest program. To induce the morphological changes, a very short hormonal treatment (as short as $2 \mathrm{~h}$ ) is sufficient [9]. It appears, therefore, that, at least in these SK-ER3 cells, the estrogen-dependent morphological differentiation program initiates independently and at an earlier time than that one leading to proliferation blockage. Interestingly, the morphological differentiation parallels the appearance of a number of biochemical markers (e.g., synaptophysin and Tau) [9] and the acquisition of the capability to synthesize, store and release a specific neurotransmitter (dopamine) [1]. This demonstrates that the estrogen-treated cells develop towards the phenotype of a mature, postmitotic neuron. Because other studies done in neural cells in culture or in vivo have demonstrated that estrogen can indeed induce the synthesis of Tau and synaptophysin and that there is a strong functional link between ER-expressing
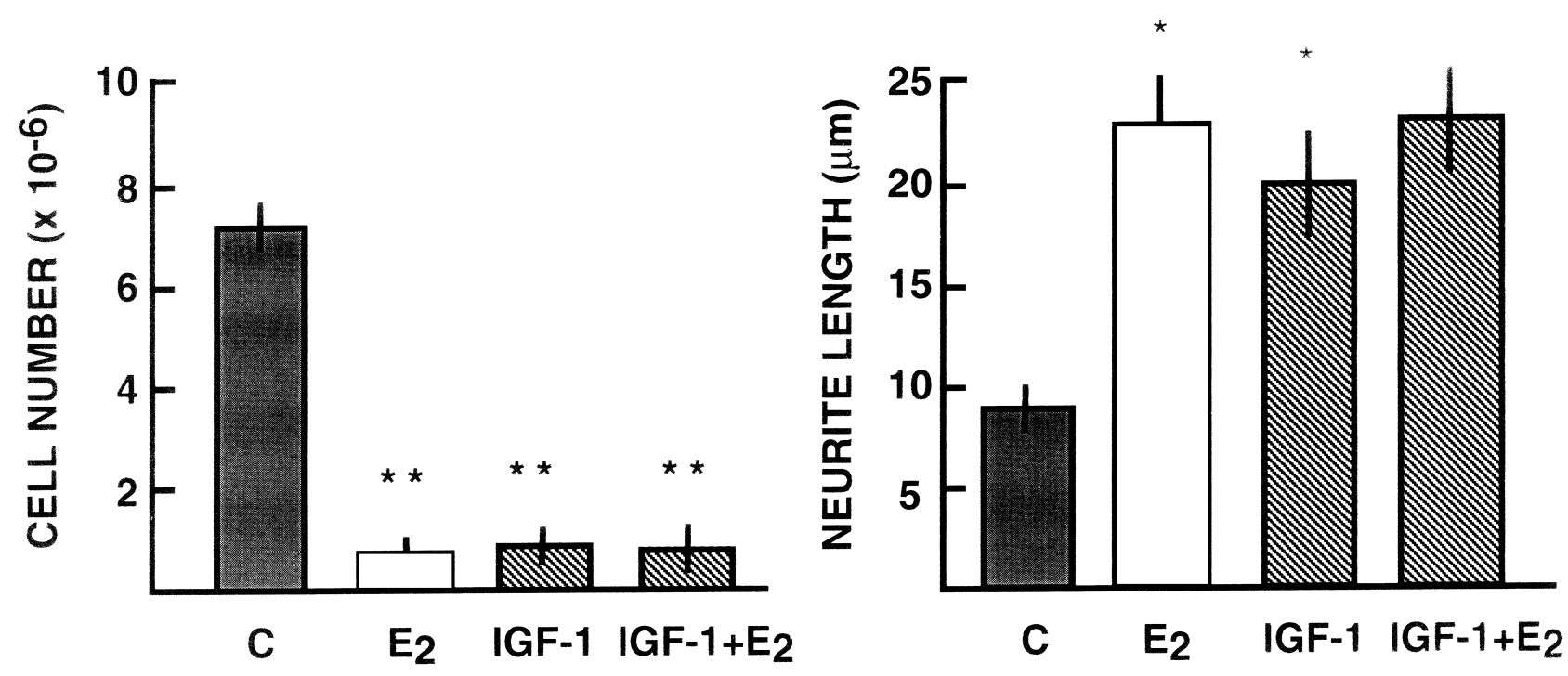

FIG. 2. Effect of IGF-I treatment on SK-ER3 morphology and growth. SK-ER3 cell were treated with IGF-I (10 nM) solubilized in the estrogen-deprived medium. IGF-I $(10 \mathrm{nM})$ was added every $48 \mathrm{~h}$ time. After 6 days in culture, the cells were counted (left panel) and neurites measured (right panel) as described in the methodology section. $* p \leq 0.01$ vs. control; ** $p \leq 0.001$ vs. control. 


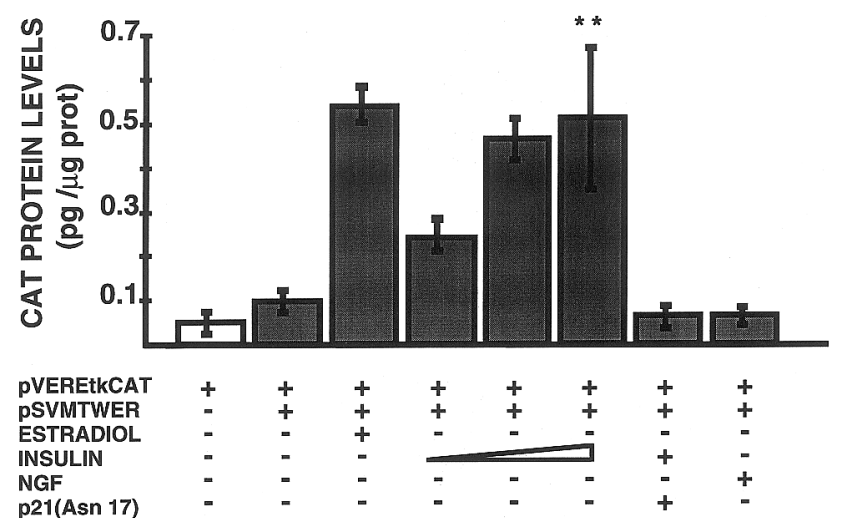

FIG. 3. Activation of ER transcriptional activity by IGF-I. Cotransfection assay in SK-N-BE cells. After calcium phosphate-mediated transfection of the reporter gene and the expression plasmid containing ER, the cells were treated as specified in the figure for $48 \mathrm{~h}$, then killed for the preparation of the extracts for the CAT quantitation. The concentrations of insulin utilized were $0.1,1$, and $10 \mu \mathrm{M}$. NGF concentration was $10 \mu \mathrm{M}$. $* p \leq 0.05$ and $* * p \leq 0.001$ vs. control.

and dopaminergic neurons, the effects observed in our model system might recapitulate what occurs in neural cells in vivo. We, therefore, postulated that also the proliferative and differentiating effects reported for SK-ER3 could mimic what is occurring during the development of the central nervous system.

\section{Cross-talk Between ER and IGF-1R Leads to Differentiation of SK-ER3 Cells}

Figure 1 shows that, even if grown in the absence of estrogens, SK-ER3 cells have a morphology that differs from the parental cell line: in having a more elongated cell body, longer neurites and having lost the tendency to grow in clusters, characteristic of the SK-N-BE cells. In a series of studies aimed at understanding the causes of this differentiated phenotype, we discovered that IGF-I can substitute for estradiol in the activation of the ER [10]. In fact, treatment of SK-ER3 cells with insulin or IGF-1 causes elongation of the neurites and growth arrest (Fig. 2). The effect on growth is of particular interest because IGF-1 has a proliferative activity in the parental SK-N-BE cell line (data not shown).

To prove that the effect of IGF-1 is indeed carried out through the ER, we performed a series of studies on the transcriptional activation of ER using the cotransfection assay in which the ER was transfected in SK-N-BE cells together with a reporter gene constituted by a basal thymidine kinase promoter with the estrogen responsive element driving the gene coding for chloramphenicol acetyltranferase (CAT). In this assay we could prove that the unliganded ER could be transcriptionally activated by treatment of the cells with IGF-1 (not shown) or insulin at high concentration. The extent of transcriptional activation of the ER induced by insulin or IGF-1 was dependent on the concentration of these

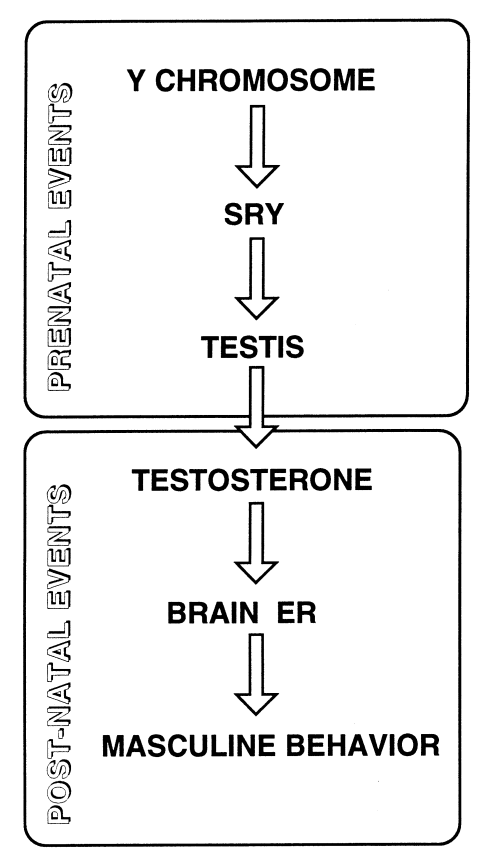

FIG. 4. Schematic view of the potential mechanisms regulating brain sexual differentiation. The current hypothesis of estrogen-dependent brain sexual differentiation depicted in the left panel might be revised in light of the potential activation of unliganded ERs by growth factors. ER activated by hormones binding to membrane receptors might have a role in the maturation of selected neurons in the earliest stages of brain development. 
hormones in the medium. Moreover, we demonstrated that this effect was linked to the activation of a specific signal transduction pathway requiring the presence of activated ras [14]. This was proved by the use of a dominant negative mutant of ras (the p21 Asn17). As shown in Fig. 3, the presence of p21 (Asn17) completely blocked the insulin-dependent activation of ER. Interestingly, NGF at a concentration as high as $10 \mu \mathrm{M}$ did not influence the ER capability to induce CAT transcription. This effect appeared to be specific for IGF-1 because treatment of these cells with NGF did not result in ER activation (Fig. 3).

\section{DISCUSSION}

The model system SK-ER3 appears to represent an interesting tool for the study of estrogen-mediated differentiation of neural cells. Other authors have utilized stable transfection for the construction of cells of neural origin expressing the ER [8]. Interestingly, the cell system generated by those authors does not display the breadth of response to estrogens shown with SK-ER3 cells. We believe that the reason for this resides in the choice of the parental cell line: Lusting et al. [8], in fact, transfected with ER the PC12 cell line, which is a phaeochromocytoma instead of a neuroblastoma cell line [8]. It is therefore likely that the phaeochromocytoma cells have reached a stage of differentiation in which certain genes cannot be activated by estrogens anymore and, therefore, the $\mathrm{E}_{2}$ differentiating potential cannot be fully expressed.

The findings here reported with regard to the effect of estrogens on SK-ER3 cells might prompt us to reconsider the current hypothesis on the activity of this sex hormone and its cognate receptor in the developing nervous system. If IGF-1 indeed activates the unliganded estrogen receptor in vivo, we may hypothesize that this growth factor, synthesized at relatively high concentration by the developing brain, induces the terminal differentiation of a subset of neuroblast expressing the ER in brain areas (and in the peripheral nervous system) that are not commonly considered as sexually dimorphic (e.g., hippocampus). It remains to be established whether this effect mediated by IGF-I is important for the development of neurons that might be endowed of sex-specific activity or not. On this line of thoughts a study on the expression of IGF-I and its cognate receptor in relation to the embryo sex is quite interesting. Male mice carrying null mutations of the genes encoding IGF-I/IGF-I-R do not display mating behavior $[3,7]$. However, it should be underlined that this deficiency could be due to the low levels of serum testosterone of these mice. The finding that multiple signals can activate the ER compels us to revise the view of the role of this receptor in the development of the CNS that might be far less schematic than previously thought (Fig. 4).. We, therefore, propose that unliganded ER can be activated by IGF-1 or insulin very early during the differentiation of the nervous system to direct the differentiation of neural cells precursors; on the basis of this hypothesis we believe that the brain areas where differentiation is controlled by ER are much more numerous than previously believed and are certainly not restricted to those areas that are involved in the control of sex-related functions.

We are well aware of the fact that the crosscoupling between ER and IGF 1R described in the present study could be linked to the specific model system utilized, and more investigations are required to prove that such a functional coupling also occurs in the developing neural cells. Yet the SK-ER3 models system might represent a unique tool for the initial dissection of the molecular events triggered in response to estrogens in cell of neural origin.

\section{ACKNOWLEDGEMENTS}

The authors wish to express their gratitude to Simona Bennici and Monica Rebecchi for their secretarial and technical contribution. These studies have been supported by the European Economic Community (BRIDGE Program BIOT-CT92) by the Italian National Council of Research (Strategic Project Antisense Oligonucleotides), and by the Italian Association for Cancer Research (AIRC).

\section{REFERENCES}

1. Agrati P.; Ma Z. Q.; Patrone C.; Picotti G. B.; Bottone M. G.; Pellicciari C.; Maggi A. Dopaminergic phenotype induced by estrogens in human neuroblastoma cell line. European J. Neurosci. 7:10071016; 1997

2. Arnold A. P.; Gorski R. A. Gonadal steroid induction of structural sex differences in the central nervous system. Annu. Rev. Neurosci. 7: 413-422; 1984.

3. Baker, J.; Hardy, M. P.; Zhou, J.; Bondy, C.; Lupu, F.; Bellvé, A. R.; Esfradiatis, A. Effects of an Igf1 gene null mutation on mouse reproduction. Mol. Endocrinol. 10:903-919; 1996.

4. Chowen, J. A.; Torres-Aleman, I.; Garcia-Segura, L. M. Trophic effects of estradiol on fetal rat hypothalamic neurons. Neuroendocrinology 56:895-901; 1992.

5. Gorski, R. A.; Gordon, J. H.; Shryne, J. F.; Southam, A. M. Evidence for a morphological sex difference within the medial preoptic area of the rat brain. Brain Res. 148:333-346; 1978.

6. Keefer, D.; Holderegger, C. The ontogeny of estrogen receptors: Brain and pituitary Dev. Brain Res. 19:183-194; 1985.

7. Liu, J. P.; Baker, J.; Perkins, A. S.; Robertson, E. J.; Esfradiatis, A. Mice carrying null mutations of the genes encoding insulin-like growth factor I (Igf-1) and type 1 IGF receptor (Igf1r). Cell 75:59-72; 1993 .

8. Lustig, R. H.; Hua, P.; Yu, W.; Ahmad, F. J.; Baas, P. An in vitro model for the effects of estrogen on neurons employing estrogen receptor-transfected PC12 cells. J. Neurosci. 14:3945-3057; 1994.

9. Ma, Z. Q.; Spreafico, E.; Pollio, G.; Santagati, S.; Cattaneo, E.; Maggi, A. Activated estrogen receptor mediates growth arrest and differentiation of a neuroblastoma cell line. Proc. Natl. Acad. Sci. USA 90: 3740-3744; 1993.

10. Ma, Z. Q.; Santagati, S.; Patrone, C.; Pollio, G.; Vegeto, E.; Maggi, A. Insulin-like growth factors activate estrogen receptor to control the growth and differentiation of the human neuroblastoma cell line SKER3. Mol. Endocrinol. 8:910-918; 1994.

11. MacLusky, N. J.; Lieberburg, I.; McEwen, B. S. The development of estrogen receptor systems in the rat brain: Perinatal development. Brain Res. 178:129-142; 1979.

12. O'Keefe, J. A.; Handa, R. J. Transient elevation of estrogen receptors in the neonatal hippocampus. Dev. Brain Res. 57:119-127; 1990.

13. O'Malley, B. W.; McGuire, W. L.; Kohler, P. O.; Korenman, S. G. Studies on the mechanism of steroid hormone regulation of synthesis of specific proteins. Recent Prog. Horm. Res. 25:105-160; 1969.

14. Patrone, C.; Ma, Z. Q.; Pollio, G.; Agrati, P.; Parker, M. G.; Maggi, A. Cross-coupling between insulin and estrogen receptor in human neuroblastoma cells. Mol. Endocrinol. 10:499-507; 1996.

15. Raisman, G.; Field, P. M. Sexual dimorphism in the neuropil of the preoptic area of the rat and its dependence on neonatal androgen. Brain Res. 54:1-29; 1973.

16. Rasmussen, J. E.; Torres-Aleman, I.; MacLusky, N.; Naftolin, F.; Robbins, R. J. The effects of estradiol on the growth patterns of estrogen receptor-positive hypothalamic cell lines. Endocrinology 126: 235-240; 1990.

17. Sohrabji, F.; Miranda, R. S.; Toran-Allerand, D. Estrogen differentially regulates estrogen and nerve growth factor receptor mRNA in adult sensory neurons. J. Neurosci. 14:459-471; 1994.

18. Takagi, K.; Kawashima, S. Culture of rat brain preoptic area neurons: Effects of sex steroids. Int. J. Dev. Neurosci. 11:63-70; 1993.

19. Urness, D. L.; Thummel, C. S. Molecular analysis of a steroid-induced regulatory hierarchy. The Drosophila E74A protein directly regulates L71-6 transcription. EMBO J. 14:6239-6246; 1995. 